OPEN ACCESS

Edited by:

Quancai Sun,

Jiangsu University, China

Reviewed by:

Chunxue Yang,

The University of Hong Kong,

Hong Kong, SAR China

Lijun Sun,

Northwest $A$ and F University, China

*Correspondence:

Qian Wu

wuqian@hbut.edu.cn

Juan Xiao

xiaojuan209218@163.com

Specialty section: This article was submitted to

Nutrition and Food Science

Technology,

a section of the journal

Frontiers in Nutrition

Received: 01 August 2021

Accepted: 23 August 2021

Published: 22 September 2021

Citation:

Feng N, She S, Hu H, Tang S, Tan J, Wu Q and Xiao J (2021) Effects of Oligomeric Procyanidins From Lotus Seedpod on the Retrogradation

Properties of Rice Starch.

Front. Nutr. 8:751627.

doi: 10.3389/fnut.2021.751627

\section{Effects of Oligomeric Procyanidins From Lotus Seedpod on the Retrogradation Properties of Rice Starch}

\author{
Nianjie Feng ${ }^{1}$, Shaowen She ${ }^{1}$, Hengfeng $\mathrm{Hu}^{2}$, Shimiao Tang ${ }^{1}$, Jiangying $\operatorname{Tan}^{1}$, Qian $\mathrm{Wu}^{1 *}$ \\ and Juan Xiao ${ }^{3 *}$ \\ 1 Key Laboratory of Fermentation Engineering, Ministry of Education, Hubei Key Laboratory of Industrial Microbiology, \\ National "111" Center for Cellular Regulation and Molecular Pharmaceutics, Hubei Research Center of Food Fermentation \\ Engineering and Technology, Hubei University of Technology, Wuhan, China, ${ }^{2}$ J.S Corrugating Machinery Co. Ltd, Wuhan, \\ China, ${ }^{3}$ State Key Laboratory of Marine Resource Utilization in South China Sea/Ministry of Education, Key Laboratory of \\ Food Nutrition and Functional Food of Hainan Province/Engineering Research Center of Utilization of Tropical Polysaccharide \\ Resources/School of Food Science and Engineering, Hainan University, Haikou, China
}

The extent of retrogradation strongly affects certain physical and cooking properties of rice starch (RS), which are important to consumers. In this study, oligomeric procyanidins from lotus seedpod (LSOPC) was prepared and used to investigate its inhibitory effect on RS retrogradation. Various structural changes of RS during retrogradation were characterized by differential scanning calorimetry, low field nuclear magnetic resonance, X-ray diffraction, scanning electron microscopy, and Fourier transform infrared spectroscopy. The results showed LSOPC could effectively retard both short- and long-term retrogradation of RS, and its inhibitory effect was dependent on the administered concentration of LSOPC. Molecule simulation revealed the interactions of RS and LSOPC, which indicated that the competition of hydrogen bonds between RS and LSOPC was the critical factor for anti-retrogradation. This inhibitory effect and mechanism of action of LSOPC could promote its applications in the field of starch anti-retrogradation.

\section{Keywords: oligomeric procyanidins, retrogradation, rice starch, molecule simulation, hydrogen bonds}

\section{INTRODUCTION}

Rice is a common food and a basic staple for hundreds of millions of people (1). Starch is the main component of rice, comprising up to about $90 \%$ of the dry weight. Retrogradation can substantially affect the physical and cooking properties of starch in the storage $(2,3)$. It is well known that starch retrogradation can be categorized as short- and long-term. Short-term retrogradation normally occurs in the first few hours after gelatinization and is induced by the reordering and recrystallization of amylose fractions, whereas long-term retrogradation is attributed to the recrystallization of amylopectin fractions that takes place over several days or even weeks.

Several methods have been found to retard or decrease starch retrogradation, such as various modifications and specially designed additives. Extrusion and microwaving are easy-to-operate physical ways, however, the effects of anti-retrogradation are limited (4-6). Acetylation and crosslinking are used as chemical modifications, and provide strong anti-retrogradation effects (7-9). 
But they are unacceptable for food safety due to the presence of residual chemicals. As for additives, it has been widely shown that retrogradation can be inhibited to a certain degree by adding amylase, saccharides, or emulsifiers (10-12).

In addition, increased awareness of food safety has led to the development of natural food additives. Natural polyphenols have attracted attention because their non-negligible inhibiting effect on starch retrogradation. Rutin, ferulic acid, epigallocatechin gallate and tannin have already been used in research on starch retrogradation (13-16). Procyanidins (PC) are natural polyphenols widely existing in plants, which are formed by the condensation of flavanol monomers $(17,18)$. The free radical scavenging ability and antioxidant efficiency of PC have generated an enormous interest in the field of natural food additives. As such, PC may offer a new opportunity to inhibit starch retrogradation. However, the interactions between PC and starches remains unclear, and the thorough interactions investigation could provide valuable information for antiretrogradation effects of $\mathrm{PC}$.

In this study, PC was prepared from lotus seedpod. The retrogradation of rice starch with and without PC was then investigated. Various structural changes of rice starch during retrogradation were characterized by differential scanning calorimetry (DSC), low field nuclear magnetic resonance (LF-

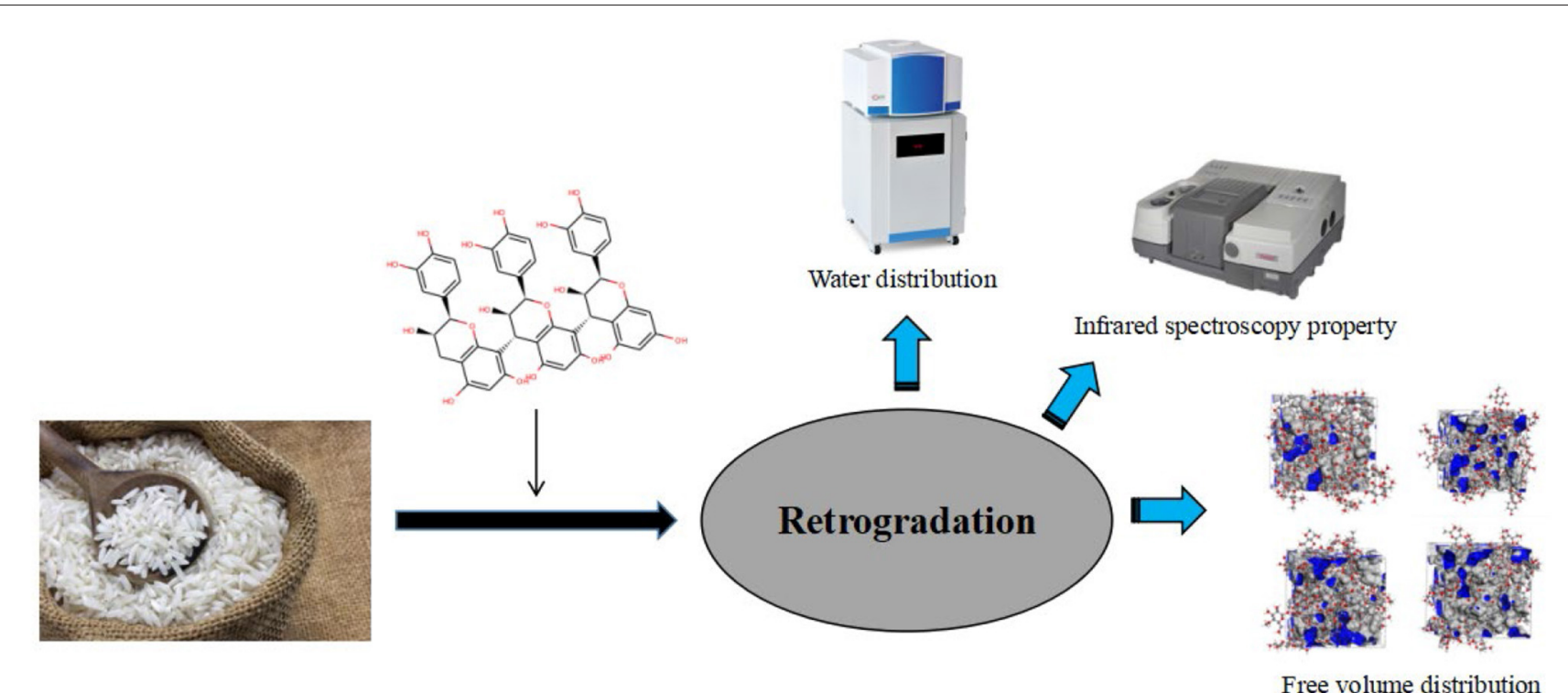

SCHEME 1 | Inhibition illustration of LSOPC on RS retrogradation.

TABLE 1 | Thermodynamic properties of RS with or without LSOPC.

\begin{tabular}{|c|c|c|c|c|}
\hline Sample & $\mathbf{T}_{\text {onset }}\left({ }^{\circ} \mathbf{C}\right)$ & $\mathbf{T}_{\text {peak }}\left({ }^{\circ} \mathbf{C}\right)$ & $\mathbf{T}_{\text {end }}\left({ }^{\circ} \mathbf{C}\right)$ & $\Delta H_{g}(\mathrm{~J} / \mathrm{g})$ \\
\hline RS & $54.37 \pm 0.13^{a}$ & $79.12 \pm 0.24^{a}$ & $103.54 \pm 0.33^{a}$ & $21.46 \pm 0.36^{a}$ \\
\hline $\mathrm{RS}+3 \% \mathrm{LSOPC}$ & $47.61 \pm 0.30^{b}$ & $70.13 \pm 0.41^{b}$ & $92.83 \pm 0.15^{\mathrm{b}}$ & $15.23 \pm 0.20^{b}$ \\
\hline RS + 6\% LSOPC & $48.22 \pm 0.52^{b}$ & $70.62 \pm 0.29^{b}$ & $93.32 \pm 0.41^{b}$ & $12.65 \pm 0.42^{c}$ \\
\hline RS + 9\% LSOPC & $40.45 \pm 0.44^{c}$ & $63.36 \pm 0.48^{c}$ & $85.61 \pm 0.23^{c}$ & $13.12 \pm 0.22^{c}$ \\
\hline
\end{tabular}

The data are given as mean \pm S.D. $(n=3)$. Different letters indicated a significant difference $(p<0.05)$.

TABLE 2 | Texture characteristic of RS with or without LSOPC.

\begin{tabular}{|c|c|c|c|c|c|}
\hline Sample & Hardness & Springiness & Cohesiveness & Gumminess & Chewiness \\
\hline RS & $0.75 \pm 0.09^{a}$ & $1.03 \pm 0.16^{a}$ & $0.76 \pm 0.03^{a}$ & $0.43 \pm 0.06^{a}$ & $0.52 \pm 0.02^{a}$ \\
\hline $\mathrm{RS}+3 \% \mathrm{LSOPC}$ & $0.64 \pm 0.08^{a}$ & $0.85 \pm 0.05^{a b}$ & $0.68 \pm 0.04^{b}$ & $0.40 \pm 0.05^{a}$ & $0.29 \pm 0.05^{b}$ \\
\hline $\mathrm{RS}+6 \% \mathrm{LSOPC}$ & $0.21 \pm 0.05^{b}$ & $0.79 \pm 0.08^{b}$ & $0.68 \pm 0.01^{b}$ & $0.14 \pm 0.04^{b}$ & $0.11 \pm 0.02^{\mathrm{c}}$ \\
\hline RS + 9\% LSOPC & $0.16 \pm 0.02^{b}$ & $0.70 \pm 0.03^{b}$ & $0.61 \pm 0.02^{c}$ & $0.11 \pm 0.04^{b}$ & $0.08 \pm 0.01^{c}$ \\
\hline
\end{tabular}

The data are given as mean $\pm S . D$. $(n=3)$. Different letters indicated a significant difference $(p<0.05)$. 
NMR), X-ray diffraction (XRD), scanning electron microscopy (SEM), Fourier transform infrared spectroscopy (FT-IR), and computer molecular simulation (Scheme 1).

\section{MATERIALS AND METHODS}

\section{Materials}

Rice starch (RS) was purchased from Wuxi Jinnong Biotechnology Co., Ltd. (Jiangsu, China). Lotus seedpods were bought from the local supermarket (Wu Zhi 2 hao).

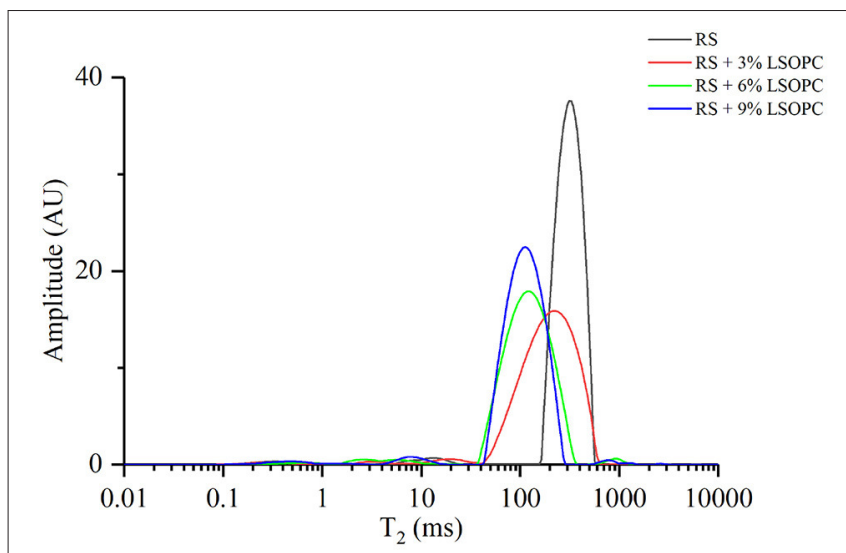

FIGURE 1 | LF-NMR spectra of RS with or without LSOPC.

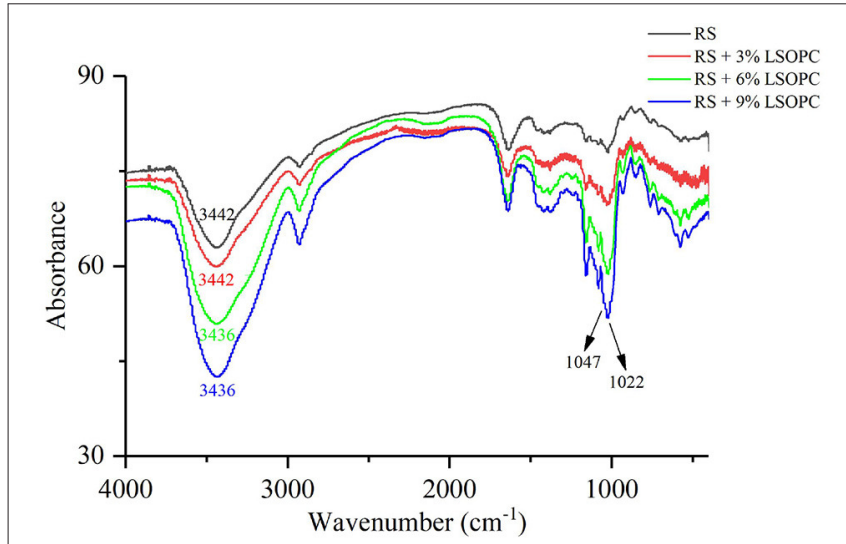

FIGURE 2 | Infrared spectroscopy spectra of RS with or without LSOPC.

TABLE 3 | Ratios of absorbance values from the infrared spectra at 1,047 and $1,022 \mathrm{~cm}^{-1}$.

\section{Sample}

RS

RS $+3 \%$ LSOPC

$\mathrm{RS}+6 \% \mathrm{LSOPC}$

$\mathrm{RS}+9 \% \mathrm{LSOPC}$
Ratios

0.915

0.908

0.852

0.843

\section{Preparation of Samples}

The method of $\mathrm{Wu}$ et al. (19) was used to prepare oligomeric procyanidins from lotus seedpod (LSOPC). The polymerization degree of LSOPC was 3. RS was mixed with LSOPC at the ratios of 100:0, 100:3, 100:6 and 100:9 (RS/LSOPC, w/w). The mixtures were added to deionized water to ensure uniformity, and the mass concentration of RS was $60 \mathrm{~g} / \mathrm{L}$.

\section{Measurement of Thermodynamic Property}

A DSC 214 (NETZSCH, Germany) was used to measure the thermodynamic properties of RS with or without LSOPC. The sample $(2 \mathrm{mg}$ ) was used, and the heating rate is programmed at $10^{\circ} \mathrm{C} / \mathrm{min}$ from 20 to $100^{\circ} \mathrm{C}$. After that, the onset temperature $\left(\mathrm{T}_{\text {onset }}\right)$, peak temperature $\left(\mathrm{T}_{\text {peak }}\right)$ and end temperature $\left(\mathrm{T}_{\text {end }}\right)$ were obtained from the pasting curve. The pasting enthalpy $\left(\Delta \mathrm{H}_{\mathrm{g}}\right)$ was analyzed by the calculation of peak area.

\section{Detection of Texture Characteristic}

After gelatinization, all samples were stored at $4^{\circ} \mathrm{C}$ for 7 days. The texture characteristics were detected by a TA-XY2i texture analyzer (Stable Micro System Co., United States). The test conditions were as follows: the probe was $\mathrm{P} / 0.5$ type, the pre-test, test and post-test speed were all $1.0 \mathrm{~mm} / \mathrm{s}$, the trigger force was set to $5.0 \mathrm{~g}$, and the shape variable was set to $40 \%$. TPA software was used to analyze the final results.

\section{LF-NMR Analysis}

Gelatinized samples were stored at $4^{\circ} \mathrm{C}$ for 7 days. All samples were transferred to the NMR tubes and a NMI 20X NMR imaging analyzer (Shanghai Niumag Co., China) was used to determine the water distribution. The treatment conditions were as follows: the number of sample point was set to 1,024 , the number of repeated scans was set to 8 , and the relaxation attenuation time was set to $2,000 \mathrm{~ms}$. CPMG pulse sequence was applied to measure the relaxation time.

\section{FT-IR Analysis}

The samples were analyzed by a Nexus 470 FT-IR spectrometer (Nicolet, United States) according to the previous method (20) with some modifications. The samples were freeze-dried, fully

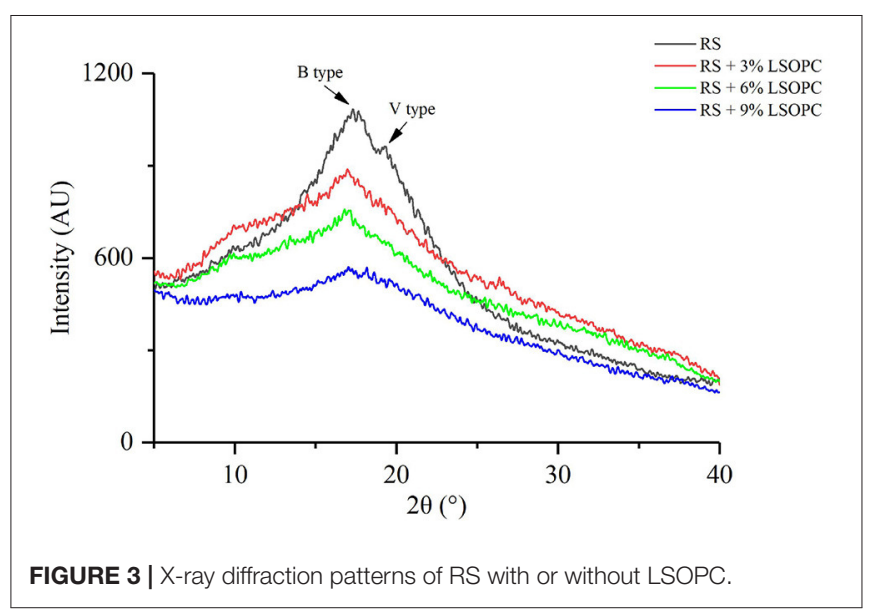


ground, and mixed with dried $\mathrm{KBr}$ powder. The wavenumber range was $4,000-400 \mathrm{~cm}^{-1}$ and the final results were analyzed by omnic 8.0 software.

\section{XRD Analysis}

The crystal structure of the freeze-dried powder sample (10 mg) was measured by a D8-Advance diffractometer (Bruker, United States). The scanning range $(2 \theta)$ was $5^{\circ}-40^{\circ}$ and the scanning speed was $2^{\circ} / \mathrm{min}$. MDI jade 6.0 software was used to analyze the final results.

\section{SEM Analysis}

About $2 \mathrm{mg}$ of freeze-dried sample was fixed and sprayed gold. Then the sample was observed by a SU-8010 scanning electron microscope (Hitachi, Japan).

\section{Determination of Dynamic Rheological Property}

All samples were cooled to room temperature after gelatinization. A DHR-3 rotational rheometer (TA Instruments Inc., United States) was applied to determine the dynamic rheological properties of samples. The test conditions were as follows: the plate diameter was $40 \mathrm{~mm}$, the gap was $0.5 \mathrm{~mm}$, the temperature was set to $25^{\circ} \mathrm{C}$, the scanning strain was set to $1 \%$, and the frequency was $0.1-10 \mathrm{~Hz}$. Finally, the spectra of storage modulus (G') and loss modulus ( $\left.G^{\prime \prime}\right)$ were obtained.

\section{Molecule Simulation}

The structural models of RS and procyanidins were downloaded from the National Center for Biotechnology Information (NCBI) using PubChem SID: 135332954 and 374367001. ChemOffice
2010 was used to optimize these molecular structures. After that, Materials Studio 8.0 was used to simulate the interactions between RS and procyanidins.

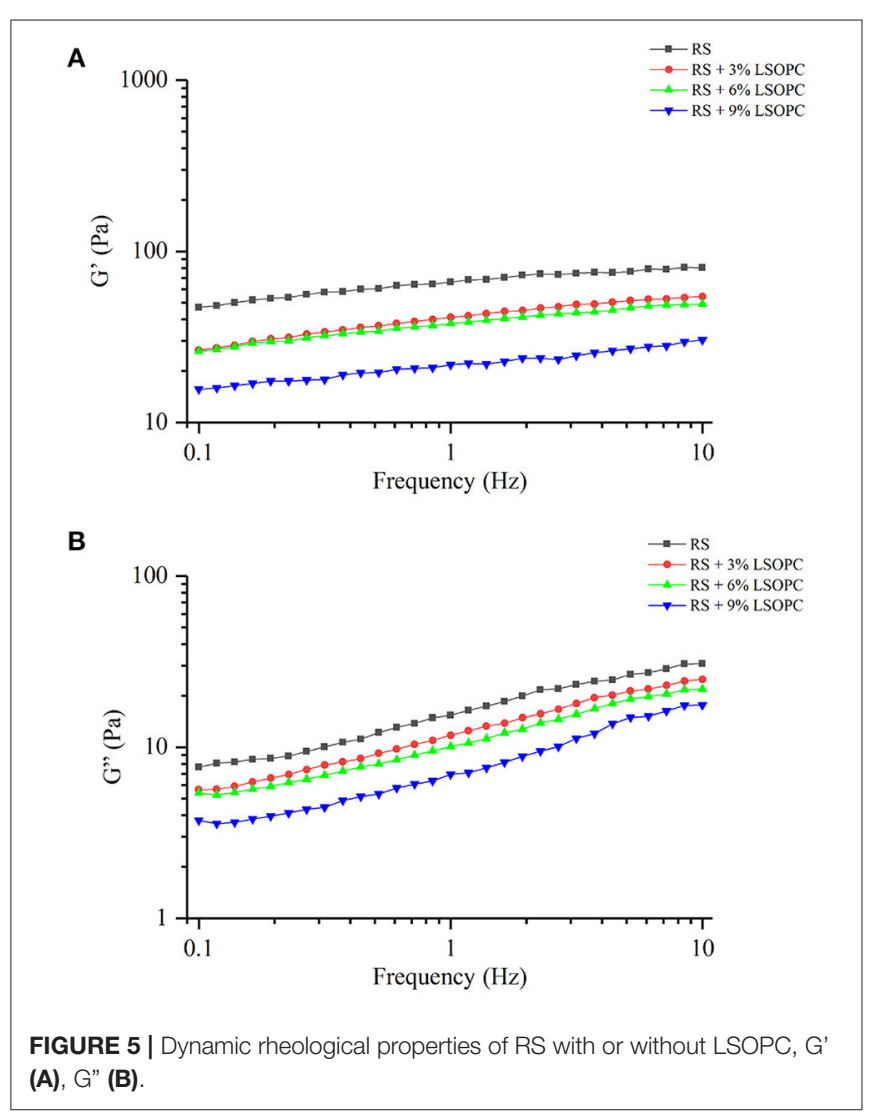

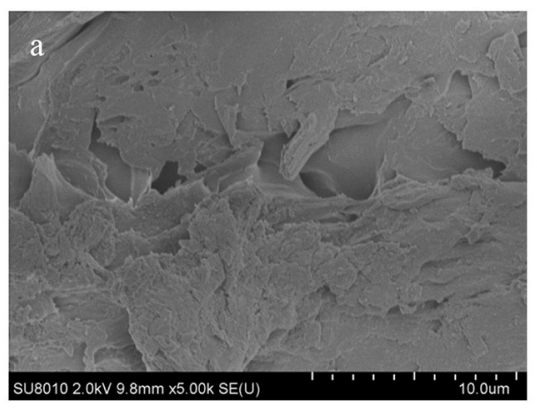
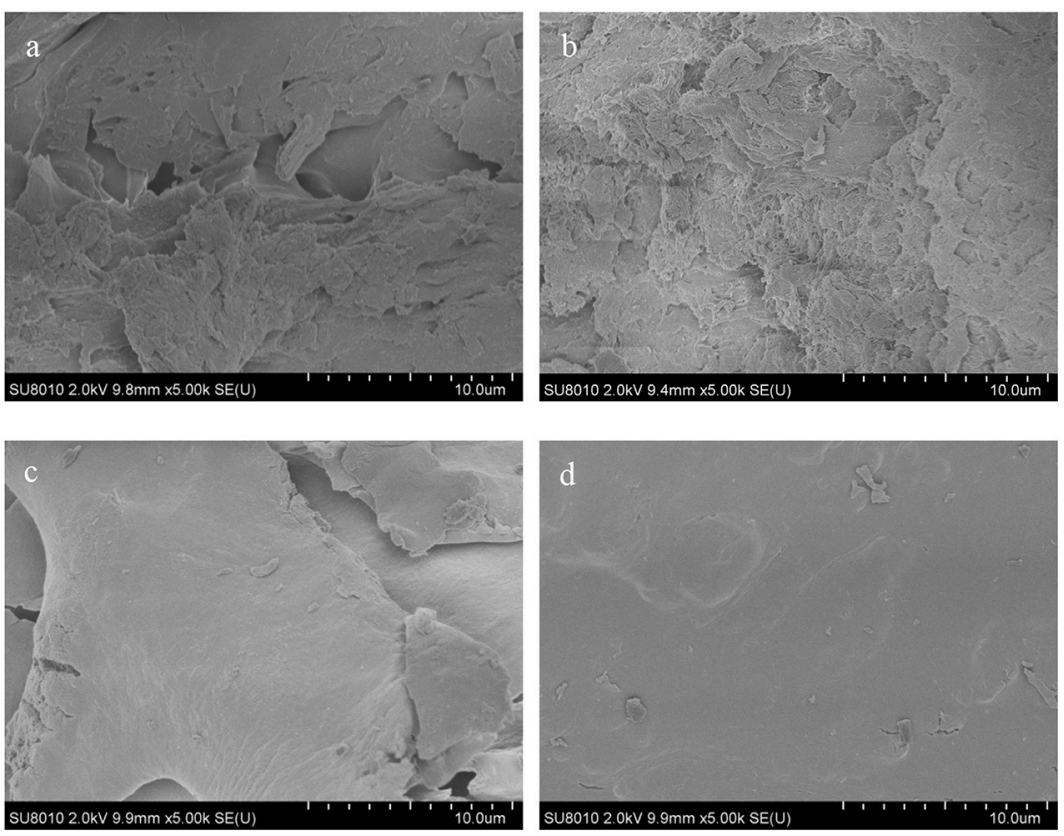

FIGURE 4 | Scanning electron micrographs of RS with or without LSOPC, RS (A), RS + 3\% LSOPC (B), RS +6\% LSOPC (C), and RS + 9\% LSOPC (D) 
A
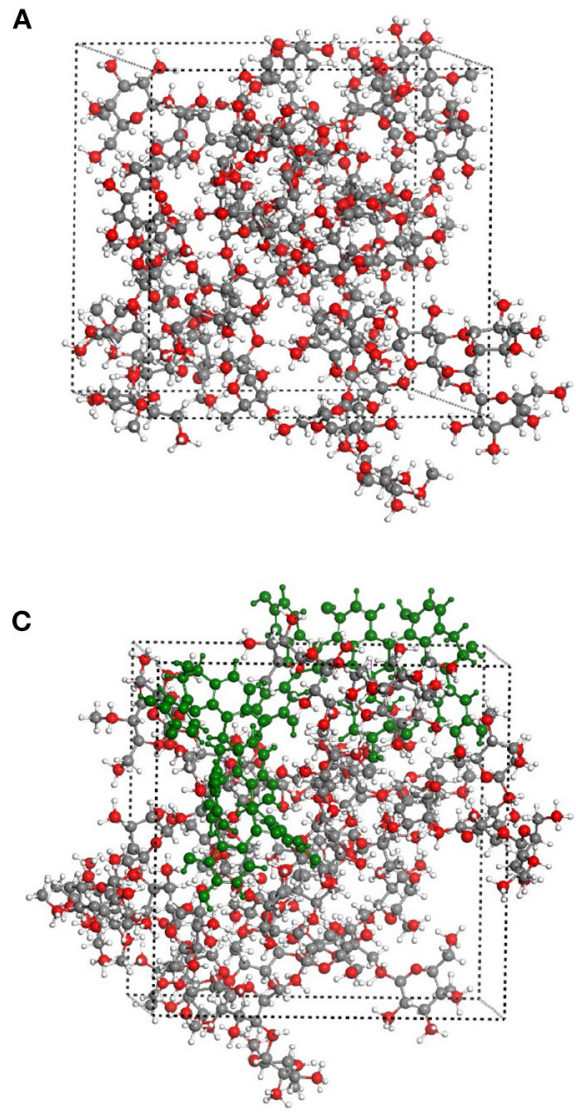

B

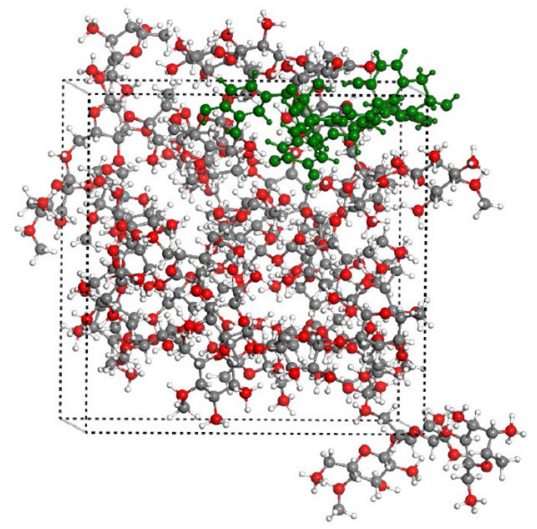

D

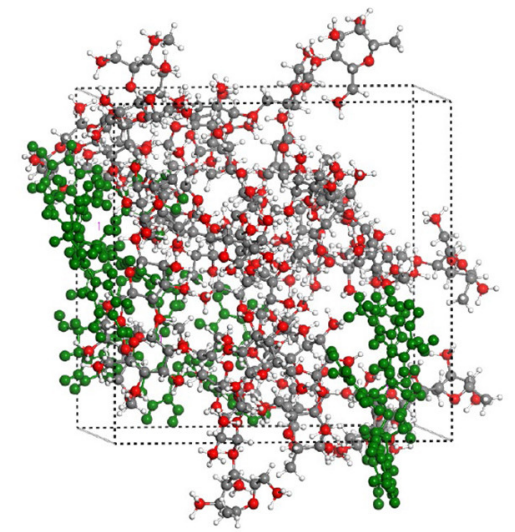

FIGURE 6 | Simulation systems of molecule of RS with or without LSOPC, OPC S (A), 1PC S (B), 2PC S (C), and 3PC S (D).

\section{Statistical Analysis}

All data were presented as means \pm standard deviation (means \pm S.D.). Analyses of variance (ANOVA) were performed by SPSS 25.0. The graph was drawn by OriginPro 8.0.

\section{RESULTS AND DISCUSSION Effect of LSOPC on the Thermodynamic Property of RS}

The thermodynamic properties are shown in Table 1. Compared with RS without LSOPC, the $\mathrm{T}_{\text {onset }}, \mathrm{T}_{\text {peak }}, \mathrm{T}_{\text {end }}$ and $\Delta \mathrm{H}_{\mathrm{g}}$ of RS with LSOPC trended downward, and the decline correlated positively to the amount of LSOPC added. This result indicated that RS with LSOPC could gelatinize at a lower temperature, which may be due to the hydroxyl group in procyanidins. The hydroxyl group, which has strong hydrophilicity, could have interacted with the side chain of the starch and then combined with its non-crystalline region. Therefore, the degree of crystallinity changed, and the energy required for starch gelatinization was reduced (21).

\section{Effect of LSOPC on the Texture Characteristic of RS}

Qiu et al. (22) found the increase of hardness was a typical retrogradation behavior of glutinous rice. As shown in Table 2, the addition of LSOPC decreased the hardness of RS gel, and the decline was positively related to the amount of LSOPC added. It indicated that procyanidins could inhibit RS retrogradation to a certain degree. A possible reason for this was that procyanidins lessened the recrystallization level of amylopectin, and thus reduced the stiffness of the starch granule and delayed the brittle hardening of mechanical properties of the macroscopical system.

\section{LF-NMR Properties of RS With or Without LSOPC}

The changes in water distribution of samples were determined by using LF-NMR analysis, and the corresponding spectra were shown in Figure 1. The length of relaxation time was inversely associated with the degree of tightness of the combination of water and matter (23). A long relaxation time indicated the proton had a higher degree of freedom and was easily discharged. The condition of the proton with a short relaxation time was the opposite. Hence the migration rules of water in RS could be analyzed according to the changes of relaxation time. Generally, the relaxation time could be divided into three periods: $T_{21}$ $(0.02-10 \mathrm{~ms})$ corresponded to the binding water, which bound the matter most tightly; $\mathrm{T}_{22}(17-165 \mathrm{~ms})$ matched the water that did not flow easily, which was between the binding water and the free water; and $\mathrm{T}_{23}(180-1,400 \mathrm{~ms})$ corresponded to the free water, which possessed the highest degree of fluidity. Figure 1 showed the amplitude of RS without LSOPC was the largest at $\mathrm{T}_{23}$, indicating the internal water was mainly free water. 
A

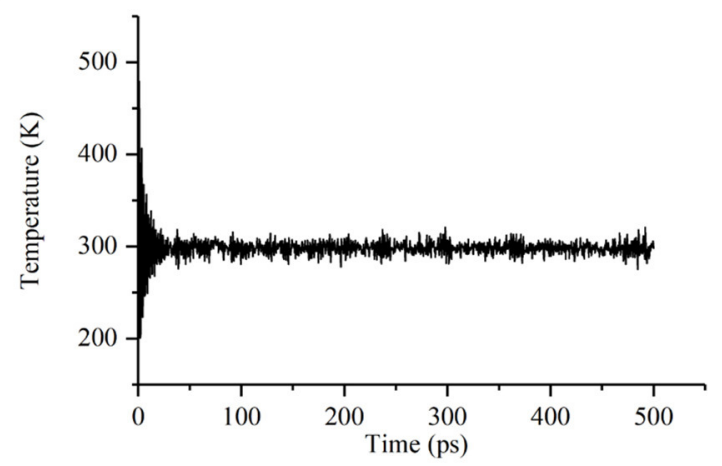

C

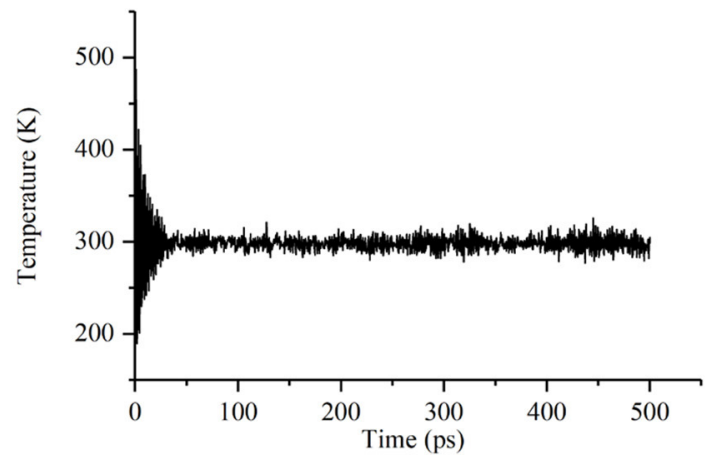

E

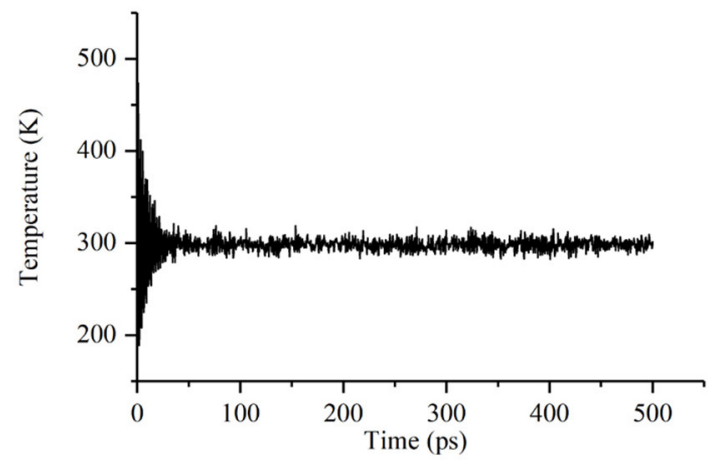

G

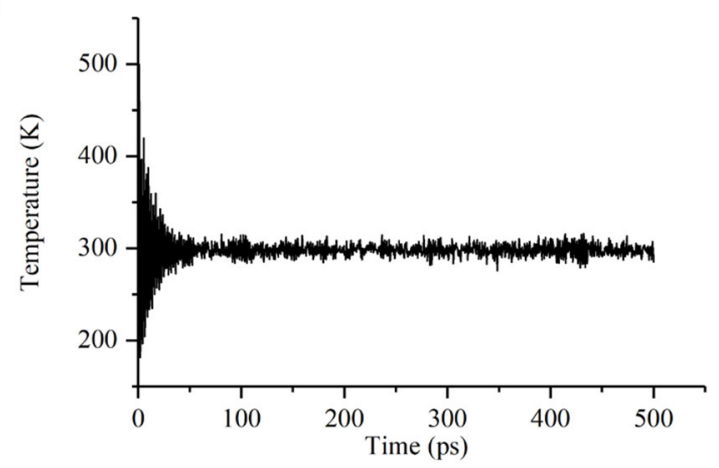

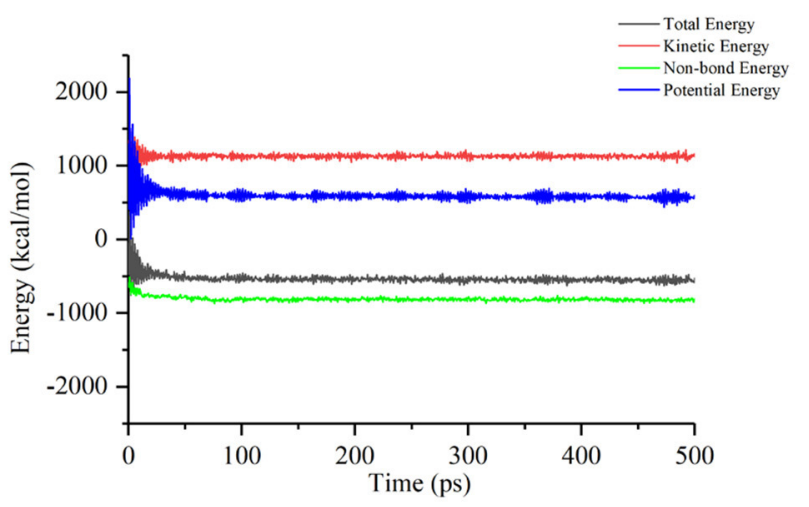

D

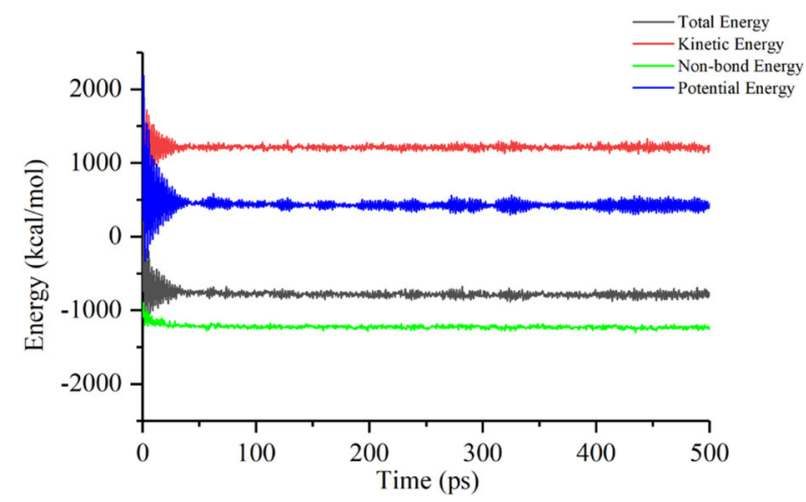

$\mathbf{F}$

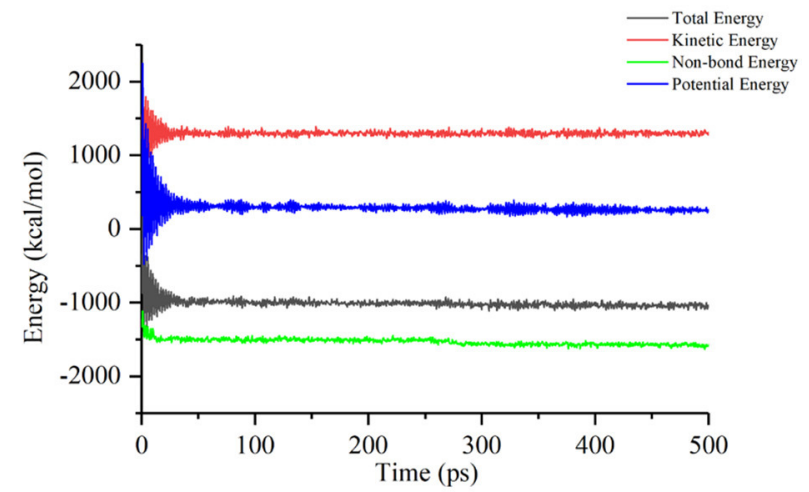

H

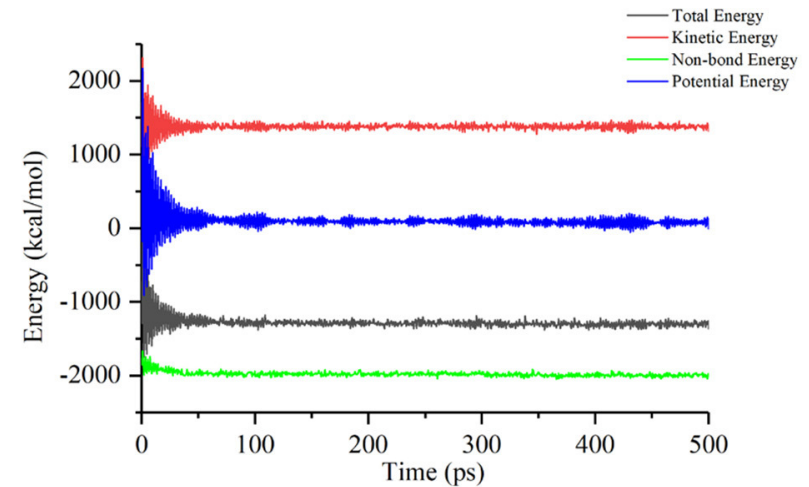

FIGURE 7 | Changes of temperature and energy with time in simulation systems, temperature change of OPC S (A), energy change of OPC S (B), temperature change of $1 P C S(C)$, energy change of $1 P C S(\mathbf{D})$, temperature change of 2PC $S(\mathbf{E})$, energy change of 2PC $S(\mathbf{F})$, temperature change of $3 P C S(\mathbf{G})$, and energy change of $3 P C S(\mathbf{H})$. 


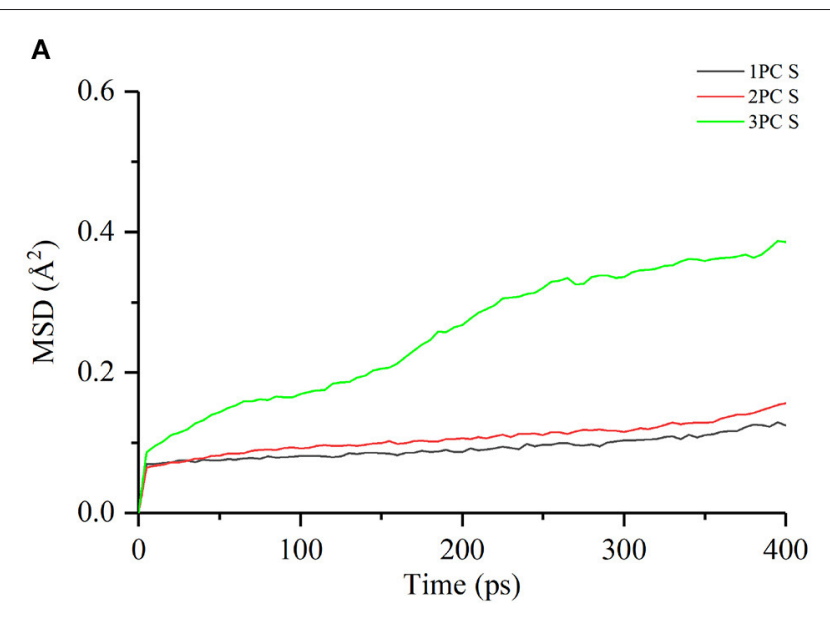

B

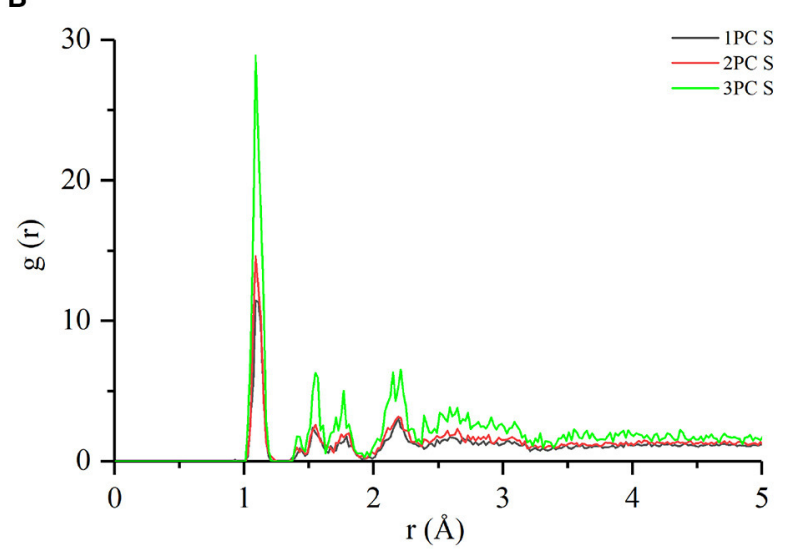

FIGURE 8 | Mean square displacement (A) and radial distribution function (B) of RS with or without LSOPC.

With the increase of LSOPC addition, the wave peak shifted to the left and finally located at $\mathrm{T}_{22}$, suggesting the water flow became harder. The results of LF-NMR showed procyanidins could increase the water holding capacity of RS, and subsequently hinder its recrystallization.

\section{FT-IR Properties of RS With or Without LSOPC}

The infrared spectra of samples are presented in Figure 2, and there was an absorption peak at $3,442 \mathrm{~cm}^{-1}$ for RS. Notably, the absorption peak did not change significantly after adding $3 \%$ LSOPC, but moved toward the direction of low wavenumber after adding 6 or $9 \%$ LSOPC. The band of 3,100-3,500 $\mathrm{cm}^{-1}$ in the infrared spectrum could reflect the hydrogen bond strength formed by intramolecular hydroxyl groups: the stronger the hydrogen bond strength, the larger the shift of the spectral band to the lower wavenumber $(24,25)$. It demonstrated that a higher proportion of procyanidins could increase the hydrogen bond strength of the system. Retrogradation is a process in which starch molecules rearrange and combine to become orderly after gelatinization. The procyanidins would interact with the starch's molecular chain to strengthen the hydrogen bonds of system and make starch retrogradation more difficult. In addition, the band of $1,047 \mathrm{~cm}^{-1}$ correlated with the orderly area of the starch, while the band of $1,022 \mathrm{~cm}^{-1}$ related to the amorphous area of the starch. The ratios of absorbance values from the infrared spectra at 1,047 and $1,022 \mathrm{~cm}^{-1}$ could reflect the orderly degree of the crystal region (26). As shown in Table 3, the ratio decreased after adding 6 or $9 \%$ LSOPC. These results indicated a higher proportion of procyanidins performed better at inhibiting the retrogradation of RS.

\section{XRD Properties of RS With or Without LSOPC}

The crystal properties of samples were measured by using XRD analysis (Figure 3). The diffraction peaks of RS appeared at $17^{\circ}$ and $20^{\circ}$. The former represented the B type crystallization of starch, and the latter reflected the $\mathrm{V}$ type crystallization of starch. The generation of a $\mathrm{V}$ type crystal might be due to the formation of the complex between amylose and lipid (27). Nevertheless, the diffraction peak intensity of $\mathrm{RS}$ at $17^{\circ}$ decreased after the addition of LSOPC, and the decreasing range rose with an increase of the LSOPC addition. The hydroxyl group, which was introduced by procyanidins, could have interacted with the molecular chain of the starch through hydrogen bonds (28). Therefore, procyanidins hampered the movement of water molecules near starch, lessened the number of water molecules that effectively participated in the retrogradation, and reduced the crystallinity of the starch.

\section{SEM Properties of RS With or Without LSOPC}

The scanning images of RS with or without LSOPC are displayed in Figure 4. There were many starch fragments with different shapes piled together, and no obvious change on starch surface occurred after adding 3\% LSOPC. However, starch fragments were reduced and became smaller with the addition of $6 \%$ LSOPC. It indicated procyanidins reduced the proportion of starch fragments and enabled the whole to be flatter and more compact. Moreover, when 9\% LSOPC was added, the starch fragments were further reduced and the surface smoothness was further enhanced. The probable reason was procyanidins competed with starch granules for the nearby water molecules, resulting in the low degree of pasting completion of starch granules (29). Thereby some starch granules had compact structures, which was not beneficial to ordering of starch molecules and hindered the retrogradation.

\section{Effect of LSOPC on the Dynamic Rheological Properties of RS}

As presented in Figure 5, the effects of LSOPC on the dynamic rheological properties of RS were determined. G' was the storage modulus, representing the energy stored in the matter during deformation. It could reflect the ability to restore to the original state, and the storage modulus was positively correlated to the restoring ability. It was reported that G' was linked to the amylose aggregation in the early stage of retrogradation (30). G” was the loss modulus, representing the energy consumed by the matter 


\section{A}

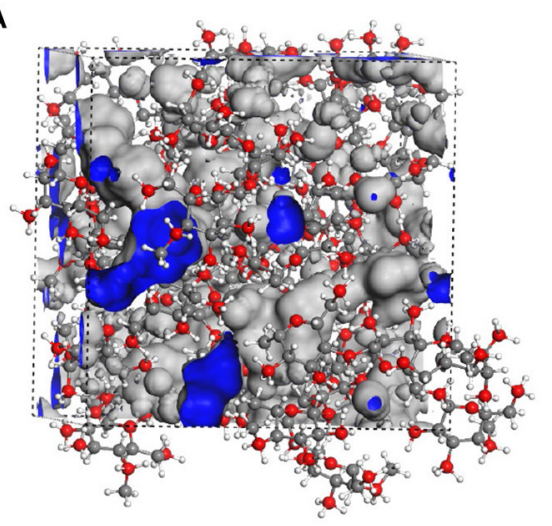

C

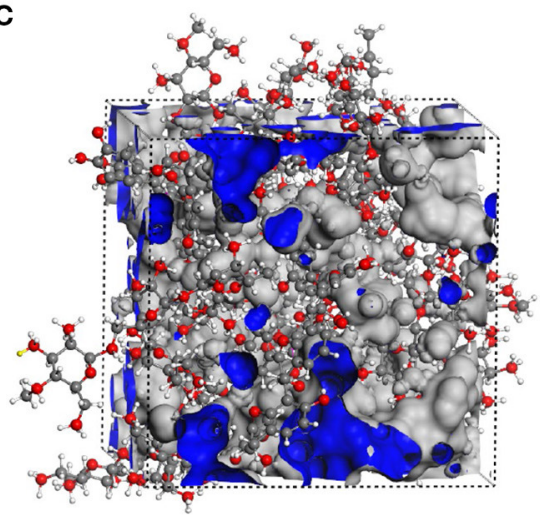

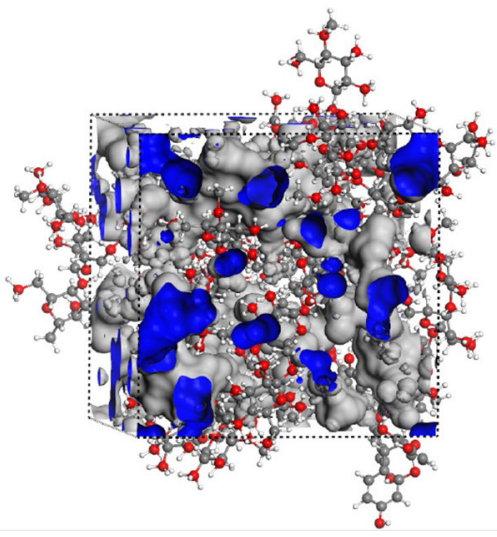

D

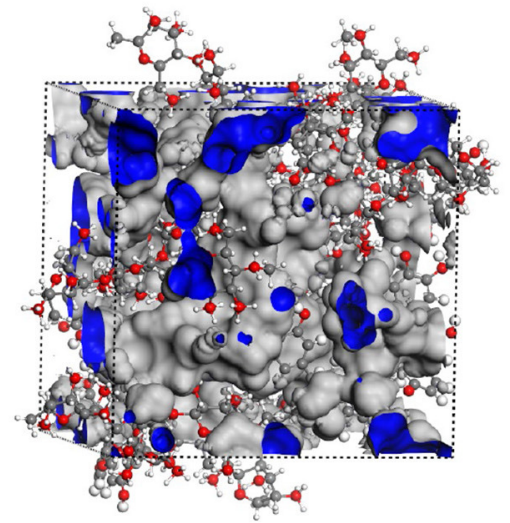

FIGURE 9 | Free volume distribution of RS with or without LSOPC, OPC S (A), 1PC S (B), 2PC S (C), and 3PC S (D).

during deformation due to the resistance of viscous drag. It could mirror the capacity to resist flow, and the loss modulus positively correlated to the resisting capacity. Figure 5 showed G' was larger than G' in all groups, and both of them tended to rise with the increase of frequency. Compared with RS, the RS-LSOPC groups revealed lower G' and G', demonstrating the dynamic moduli declined by the addition of procyanidins. A possible reason may be that procyanidins contended with the starch for the nearby water molecules, decreasing the amylose exudation and forming a weak gel structure (31). Overall, procyanidins had an anti-retrogradation effect on starch in the early stage.

\section{Simulation Study of Molecule}

The interactions between starch and procyanidins were simulated using Materials Studio 8.0. Molecular interaction volume models were shown in Figure 6. The content of procyanidins was $0 \%$ (0PC S), 3\% (1PC S), 6\% (2PC S), and 9\% (3PC S), respectively. As displayed in Figure 7, the temperature fluctuation of the four systems was not obvious after a long dynamic simulation. Besides, with the increase of the procyanidins ratio, the potential energy, non-bond energy, and total energy of the system decreased, while the kinetic energy increased slightly. It indicated that the addition of procyanidins enhanced the stability of system, but whether the movement ability was improved still needs further verification. Mean square displacement refers to the degree that the spatial position of molecules in the simulation system deviates from the initial position at a certain time, which is used to characterize the movement ability of a molecular chain (32, 33). As exhibited in Figure 8A, the mean square displacement of the system increased over time, and the increment was proportional to the ratio of procyanidins. It showed the addition of procyanidins promoted the movement ability of the starch chain. Radial distribution function is employed to describe the molecular dynamics of the system. The probability of finding a particle at a certain distance around another particle can be obtained by this function. The radial distribution function is helpful in describing the structure of the molecular system and can characterize the molecular interactions of a simulation system (34). The peak intensity comparison of $1.1 \AA$ was 3PC $S>2$ PC $S>1$ PC $S$ (Figure 8B), which implied the higher the content of procyanidins, the stronger the interaction force. It was consistent with the results of FT-IR analysis. According to the theory of free volume, the volume of a solid or liquid includes occupied volume and free volume. The former is the volume occupied by molecules themselves, and the latter is the space between molecules. Free volume is distributed in the polymer irregularly, which provides the active space for 
molecules. Notably, the free volume can significantly affect the movement ability of a polymer (35). The free volume distribution of 0PC S, 1PC S, 2PC S, and 3PC S are presented in Figure 9, the percentage was $26.71,35.77,39.97$, and $41.78 \%$, respectively. It means the movement ability of the starch chain improved as the procyanidins ratio increase, which confirmed the results of mean square displacement.

\section{CONCLUSION}

This study researched the effects of LSOPC on the retrogradation of RS. The results of texture characteristics showed LSOPC could reduce the degree of retrogradation of starch. DSC analysis indicated LSOPC were able to decrease the energy required for starch gelatinization. The reordering and recrystallization processes of starch molecules were effectively hampered by LSOPC, which was demonstrated by FT-IR, SEM, LF-NMR, and XRD analyses. The formation of a weak gel structure between LSOPC and RS was illustrated by the analysis of the results of the dynamic rheological properties. Molecule simulation implied LSOPC could enhance the stability of system and the movement ability of the starch chain. In short, the retrogradation of RS could be obviously retarded, and the effects were positively correlated with the procyanidins ratio. The study shows that procyanidins could be useful for the anti-retrogradation of rice products.

\section{REFERENCES}

1. Ali N. Co-occurrence of citrinin and ochratoxin a in rice in Asia and its implications for human health. J Sci Food Agric. (2018) 98:20559. doi: 10.1002/jsfa.8667

2. Ai J, Witt T, Cowin G, Dhital S, Turner MS, Stokes JR, et al. Antistaling of high-moisture starchy food: effect of hydrocolloids, emulsifiers and enzymes on mechanics of steamed-rice cakes. Food Hydrocoll. (2018) 83:454-64. doi: 10.1016/j.foodhyd.2018.05.028

3. Lian XJ, Kang HQ, Sun HB, Liu LZ, Li L. Identification of the main retrogradation-related properties of rice starch. J Agric Food Chem. (2015) 63:1562-72. doi: 10.1021/jf503203r

4. Sun HX, Ju Q, Ma J, Chen JC, Li YX, Yuan YQ, et al. The effects of extruded corn flour on rheological properties of wheat-based composite dough and the bread quality. Food Science Nutrition. (2019) 7:297785. doi: 10.1002/fsn3.1153

5. Liu YF, Chen J, Wu JY, Luo SJ, Chen RY, Liu CM, et al. Modification of retrogradation property of rice starch by improved extrusion cooking technology. Carbohydr Polym. (2019) 213:192-8. doi: 10.1016/j.carbpol.2019.02.089

6. Joyner HS, Jones KE, Rasco BA. Microwave pasteurization of cooked pasta: effect of process parameters on texture and quality for heat-and-eat and readyto-eat meals. J Food Sci. (2016) 81:E1447-56. doi: 10.1111/1750-3841.13334

7. Olagunju AI, Omoba OS, Enujiugha VN, Wiens RA, Gough KM, Aluko E, et al. Influence of acetylation on physicochemical and morphological characteristics of pigeon pea starch. Food Hydrocoll. (2020) 100:105424. doi: 10.1016/j.foodhyd.2019.105424

8. Nawaz MA, Fukai S, Prakash S, Bhandari B. Effect of starch modification in the whole white rice grains on physicochemical properties of two contrasting rice varieties. J Cereal Sci. (2018) 80:143-9. doi: 10.1016/j.jcs.2018.02.007

9. Babu AS, Naik GNM, James J, Bin Aboobacker A, Eldhose A, Mohan RJ. A comparative study on dual modification of banana (Musa paradisiaca) starch by microwave irradiation and cross-linking. J Food Meas Charact. (2018) 12:2209-17. doi: 10.1007/s11694-018-9837-x

\section{DATA AVAILABILITY STATEMENT}

The original contributions presented in the study are included in the article/supplementary material, further inquiries can be directed to the corresponding author/s.

\section{AUTHOR CONTRIBUTIONS}

NF: conceptualization, methodology, data curation, and writingoriginal draft. SS: data curation and methodology. $\mathrm{HH}$ : investigation and funding acquisition. ST: investigation and visualization. JT: data curation. QW: conceptualization, writingreview and editing, supervision, and funding acquisition. JX: supervision and funding acquisition. All authors contributed to the article and approved the submitted version.

\section{FUNDING}

This work was financially supported by National Natural Science Foundation of China (Nos. 32001705 and 21908048), State Key Laboratory of Marine Resource Utilization in South China Sea (Hainan University) (No. MRUKF2021002), Key Laboratory of Food Nutrition and Functional Food of Hainan Province (No. KF202009), and the Collaborative Grant-in-Aid of the HBUT National 111 Center for Cellular Regulation and Molecular Pharmaceutics (No. XBTK-2020005).

10. Grewal N, Faubion J, Feng GH, Kaufman RC, Wilson JD, Shi YC. Structure of waxy maize starch hydrolyzed by maltogenic $\alpha$-amylase in relation to its retrogradation. J Agric Food Chem. (2015) 63:4196201. doi: $10.1021 /$ jf506215s

11. Kim YA, Shim HR, Rho J. Effect of oligosaccharides on retrogradation of Sulgidduk. J East Asian Soc Dietary Life. (2015) 25:513-24. doi: 10.17495/easdl.2015.6.25.3.513

12. Prakaywatchara P, Wattanapairoj C, Thirathumthavorn D. Effects of emulsifier types and levels in combination with glycerol on the gelatinization and retrogradation properties of gluten-free rice-based wonton wraps. Starch. (2018) 70:1700227. doi: 10.1002/star.201700227

13. Zhu F, Wang YJ. Rheological and thermal properties of rice starch and rutin mixtures. Food Res Int. (2012) 49:757-62. doi: 10.1016/j.foodres.2012.09.031

14. Karunaratne R, Zhu F. Physicochemical interactions of maize starch with ferulic acid. Food Chem. (2016) 199:3729. doi: 10.1016/j.foodchem.2015.12.033

15. Pan JX, Li M, Zhang SK, Jiang YL, Lv YJ, Liu J, et al. Effect of epigallocatechin gallate on the gelatinisation and retrogradation of wheat starch. Food Chem. (2019) 294:209-15. doi: 10.1016/j.foodchem.2019. 05.048

16. Du J, Yao F, Zhang MY, Khalifa I, Li KK, Li CM. Effect of persimmon tannin on the physicochemical properties of maize starch with different amylose/amylopectin ratios. Int J Biol Macromol. (2019) 132:1193-9. doi: 10.1016/j.ijbiomac.2019.04.046

17. Xiao Y, Hu ZZ, Yin ZT, Zhou YM, Liu TY, Zhou XL, et al. Profiling and distribution of metabolites of procyanidin B2 in mice by UPLC-DAD-ESI-IT-TOF-MS ${ }^{\mathrm{n}}$ technique. Front Pharmacol. (2017) 8:231. doi: 10.3389/fphar.2017.00231

18. Zhou HC, Lin YM, Li YY, Li M, Wei SD, Chai WM, et al. Antioxidant properties of polymeric proanthocyanidins from fruit stones and pericarps of Litchi chinensis Sonn. Food Res Int. (2011) 44:613-20. doi: 10.1016/j.foodres.2010.12.016

19. Wu Q, Chen HY, Lv ZJ, Li SY, Hu B, Guan YF, et al. Oligomeric procyanidins of lotus seedpod inhibits the formation of advanced glycation 
end-products by scavenging reactive carbonyls. Food Chem. (2013) 138:1493502. doi: 10.1016/j.foodchem.2012.10.111

20. Wang JY, Bie M, Zhou WJ, Xie BJ, Sun Z. D. Interaction between carboxymethyl pachyman and lotus seedpod oligomeric procyanidins with superior synergistic antibacterial activity. Carbohydr Polym. (2019) 212:1120. doi: 10.1016/j.carbpol.2019.02.030

21. Zhu F, Cai YZ, Sun M, Corke H. Effect of phytochemical extracts on the pasting, thermal, and gelling properties of wheat starch. Food Chem. (2009) 112:919-23. doi: 10.1016/j.foodchem.2008.06.079

22. Qiu S, Punzalan ME, Abbaspourrad A, Padilla-Zakour OI. High water content, maltose and sodium dodecyl sulfate were effective in preventing the long-term retrogradation of glutinous rice grains-a comparative study. Food Hydrocoll. (2020) 98:105247. doi: 10.1016/j.foodhyd.2019.105247

23. Nawaz A, Xiong ZY, Xiong HG, Irshad S, Chen L, Wang PK, et al. The impact of hydrophilic emulsifiers on the physico-chemical properties, microstructure, water distribution and in vitro digestibility of proteins in fried snacks based on fish meat. Food Function. (2019) 10:6927-35. doi: 10.1039/C9FO0 $1312 \mathrm{~A}$

24. Ogawa K, Yamazaki I, Yoshimura T, Ono S, Rengakuji S, Nakamura Y, et al. Studies on the retrogradation and structural properties of waxy corn starch. Bull Chem Soc Jpn. (1998) 71:1095-100. doi: 10.1246/bcsj.71.1095

25. Kizil R, Irudayaraj J, Seetharaman K. Characterization of irradiated starches by using FT-Raman and FTIR spectroscopy. J Agric Food Chem. (2002) 50:3912-8. doi: 10.1021/jf011652p

26. Flores-Morales A, Jiménez-Estrada M, Mora-Escobed, R. Determination of the structural changes by FT-IR, Raman, and CP/MAS ${ }^{13} \mathrm{C}$ NMR spectroscopy on retrograded starch of maize tortillas. Carbohydr Polym. (2012) 87:618. doi: 10.1016/j.carbpol.2011.07.011

27. Xiao HX, Lin QL, Liu GQ, Wu Y, Tian W, Wu W, et al. Effect of green tea polyphenols on the gelatinization and retrogradation of rice starches with different amylose contents. J Med Plants Res. (2011) 5:4298-303. doi: 10.5897/JMPR.9000481

28. Guzar I, Ragaee S, Seetharaman K. Mechanism of hydrolysis of native and cooked starches from different botanical sources in the presence of tea extracts. J Food Sci. (2012) 77:C1192-6. doi: 10.1111/j.1750-3841.2012. 02929.x

29. Russell PL. The ageing of gels from starches of different amylose/amylopectin content studied by differential scanning calorimetry. J Cereal Sci. (1987) 6:147-58. doi: 10.1016/S0733-5210(87)80051-6
30. Nagano T, Tamaki E, Funami T. Influence of guar gum on granule morphologies and rheological properties of maize starch. Carbohydr Polym. (2008) 72:95-101. doi: 10.1016/j.carbpol.2007.07.028

31. Salehi M, Tabarsa M, Amraie M, Anvari M, Rezaei M, Smith BM. Characterization of rheological and structural properties of a gum from Balangu seeds. Int J Biol Macromol. (2018) 117:294-300. doi: 10.1016/j.ijbiomac.2018.05.033

32. Chen N, Chen L, Gao HX, Zeng WC. Mechanism of bridging and interfering effects of tea polyphenols on starch molecules. J Food Proc Preserv. (2020) 44:e14576. doi: 10.1111/jfpp.14576

33. Dai TT, Li RY, Liu CM, Liu W, Li T, Chen J, et al. Effect of rice glutelinresveratrol interactions on the formation and stability of emulsions: a multiphotonic spectroscopy and molecular docking study. Food Hydrocoll. (2019) 97:105234. doi: 10.1016/j.foodhyd.2019.105234

34. Mofradnia SR, Ashouri R, Tavakoli Z, Shahmoradi F, Rashedi H, Yazdian F, et al. Effect of zero-valent iron/starch nanoparticle on nitrate removal using MD simulation. Int J Biol Macromol. (2019) 121:72733. doi: 10.1016/j.ijbiomac.2018.09.183

35. Horn NR. A critical review of free volume and occupied volume calculation methods. J Memb Sci. (2016) 518:289-94. doi: 10.1016/j.memsci.2016.07.014

Conflict of Interest: The authors declare that the research was conducted in the absence of any commercial or financial relationships that could be construed as a potential conflict of interest.

Publisher's Note: All claims expressed in this article are solely those of the authors and do not necessarily represent those of their affiliated organizations, or those of the publisher, the editors and the reviewers. Any product that may be evaluated in this article, or claim that may be made by its manufacturer, is not guaranteed or endorsed by the publisher.

Copyright $\odot 2021$ Feng, She, Hu, Tang, Tan, Wu and Xiao. This is an open-access article distributed under the terms of the Creative Commons Attribution License (CC $B Y)$. The use, distribution or reproduction in other forums is permitted, provided the original author(s) and the copyright owner(s) are credited and that the original publication in this journal is cited, in accordance with accepted academic practice. No use, distribution or reproduction is permitted which does not comply with these terms. 